PHYSICAL REVIEW D 96, 019901(E) (2017)

\title{
Erratum: Tenth-order electron anomalous magnetic moment: Contribution of diagrams without closed lepton loops [Phys. Rev. D 91, 033006 (2015)]
}

Tatsumi Aoyama, Masashi Hayakawa, Toichiro Kinoshita, and Makiko Nio

(Received 4 June 2017; published 10 July 2017)

DOI: 10.1103/PhysRevD.96.019901

The tenth-order term $A_{1}^{(10)}$ given in Eq. (9) reported in our paper [1] must be replaced by

$$
A_{1}^{(10)}=6.599(223)
$$

This shift is caused primarily by the correction of a programming error in one of the integrals, X024, but also by further numerical improvement of all 389 integrals of Set V.

In order to improve the theoretical prediction for the electron anomalous magnetic moment $a_{e}$, we have carried out a new numerical evaluation of the 389 integrals of Set V, which represent 6354 Feynman vertex diagrams without lepton loops. During this work, we found that the integral X024 was given an incorrect value in Table I of [1] due to an incorrect assignment of integration variables. The 14 Feynman parameters of X024 subject to a linear constraint can be mapped onto an 11-dimensional unit hypercube, because of the presence of two self-energy subdiagrams in X024. The error was caused by the assumption that X024 has one more constraint reducing the dimension of the hypercube to 10 . The correction of this error changes the X024 integral from $-6.0902(246)$ to $-7.3516(208)$. The shift -1.26 affects the Set V contribution and, hence, the tenth-order mass-independent term $A_{1}^{(10)}$. All the other 388 integrals are free from error and consistent with the new evaluation.

Combining the new and the corrected old calculations statistically, we obtain the replacement of Eq. (8) of [1],

$$
A_{1}^{(10)}[\text { Set V }]=7.530(223)
$$

as the best estimate of the Set V contribution. The details will be given elsewhere. Including the contribution of the diagrams with fermion loops to (2), we have obtained the updated tenth-order mass-independent term, Eq. (1).

Together with the hadronic and electroweak contributions, the new QED formula leads to the theoretical prediction,

$$
a_{e} \text { (theory) }=1159652182.031(15)(15)(720) \times 10^{-12},
$$

where the uncertainties are due to the tenth-order QED (1), the hadronic contribution, and the fine-structure constant $\alpha$ from left to right. This new value of $a_{e}$ includes two other updates besides the tenth-order QED. One is the near-exact value of the eighth-order term [2],

$$
A_{1}^{(8)}=-1.912245764 \cdots
$$

The other is the fine-structure constant $\alpha$ derived from the $\mathrm{Rb}$ atom recoil measurement $h / m_{\mathrm{Rb}}$ combined with the atomic masses $A(e)$ and $A(\mathrm{Rb})$ of the electron and $\mathrm{Rb}$ atom, respectively, and the Rydberg constant $R_{\infty}[3,4]$

$$
\alpha^{-1}(\mathrm{Rb}: 2016)=137.035998996(85) \text {. }
$$

The theoretical prediction of the muon anomalous magnetic moment $\left(a_{\mu}\right)$ is also to be modified due to Eq. (2), but the shift is far smaller than the current theoretical uncertainty of $a_{\mu}$.

From the theory and the best measurement of $a_{e}[5,6]$, we obtain the inverse fine-structure constant,

$$
\alpha^{-1}\left(a_{e}: 2017\right)=137.0359991500(18)(18)(330),
$$

where the uncertainties are due to the tenth-order QED (1), the hadronic contribution, and the measurement of $a_{e}$. 


\section{ACKNOWLEDGMENTS}

We thank Dr. B. Taylor for suggesting prompt publication of this work. Numerical calculations were conducted on the supercomputing systems RICC and HOKUSAI-GreatWave, both at RIKEN. M. N. is supported in part by the JSPS Grant-in-Aid for Scientific Research (C) No. 16K05338.

[1] T. Aoyama, M. Hayakawa, T. Kinoshita, and M. Nio, Phys. Rev. D 91, 033006 (2015).

[2] S. Laporta, arXiv:1704.06996.

[3] R. Bouchendira, P. Cladé, S. Guellati-Khélifa, F. Nez, and F. Biraben, Phys. Rev. Lett. 106, 080801 (2011).

[4] P. J. Mohr, D. B. Newell, and B. N. Taylor, Rev. Mod. Phys. 88, 035009 (2016).

[5] D. Hanneke, S. Fogwell, and G. Gabrielse, Phys. Rev. Lett. 100, 120801 (2008).

[6] D. Hanneke, S. Fogwell Hoogerheide, and G. Gabrielse, Phys. Rev. A 83, 052122 (2011). 